\title{
Photonic-Assisted Microwave Frequency Doubling based on Silicon Ring Modulator
}

\author{
Haifeng Shao ${ }^{1,2}$, Xiaoqing Jiang ${ }^{1}$, Jianyi Yang ${ }^{1}$, Yingtao $\mathrm{Hu}^{2}$, Gunther Roelkens ${ }^{2}$, Hui $\mathrm{Yu}^{{ }^{1 *}}$ \\ ${ }^{1}$ Institute of Microelectronics and Optoelectronics, Department of Information Science and Electronic Engineering, Zhejiang \\ University, Hang Zhou 310027, China \\ ${ }^{2}$ Photonics Research Group, Department of Information Technology, Ghent University-imec, Center for Nano- and Biophotonics \\ (NB photonics), St-Pietersnieuwstraat 41, Gent 9000, Belgium \\ Email: huiyu@zju.edu.cn
}

\begin{abstract}
We experimentally demonstrate an integrated optical millimeter-wave signal generator based on a silicon ring modulator. A $20 \mathrm{GHz}$ microwave signal with $17 \mathrm{~dB}$ suppression ratio is obtained with a $10 \mathrm{GHz}$ input signal.
\end{abstract}

Keywords-millimeter signal generator, silicon ring modulator

\section{INTRODUCTION}

Millimeter ( $\mathrm{mm}$ ) wave signal generator is highly desirable in many applications such as radar, wireless communication radio-over-fiber (RoF) system, and so on. Since the generated $\mathrm{mm}$-wave signal is usually distributed to a remote site through the fiber, mm-wave signal generation in the optical domain is able to simplify the RoF system and reduce its cost. The frequency multiplication technique, which uses a lowfrequency RF oscillator and an electro-optical converter to generate a high-frequency RF signal, is undoubtedly a costeffective solution. Most reported techniques to multiply the frequency of the RF signal are based on Mach-Zehnder modulators (MZMs) [1-3], or phase shifters [4]. Filters are required sometimes to suppress undesired optical harmonics. Although these MZMs offer satisfactory performances for the RF frequency multiplication, their sizes are normally on the scale of centimeter in order to produce enough phase shift and modulation depth, and therefore the power consumption is quite large. In this paper, we present, for the first time to our knowledge, a frequency doubling system based on an integrated silicon carrier-depletion ring modulator. A $20 \mathrm{GHz}$ millimeter signal is successfully generated. Compared with MZMs, our scheme offers plenty of advantages such as compact footprint and ultra-low power consumption. Furthermore, the device is fabricated on silicon on insulator (SOI) platform which is CMOS compatible. Therefore, it has great potential for the low-cost, large-scale and high-density integration in the future.

\section{OPERATION PRINCIPLE}

The carrier-depletion silicon ring modulator is widely used for on-off key modulation in optical interconnect systems. Here this device is used to double the frequency of the driving RF signal by tuning the operation wavelength. Its operation principle is shortly discussed in this section. According to the time rate equation [5], the static transmitted field in the bus waveguide of an all-pass ring modulator is expressed as

$$
S_{t}=A \frac{j\left(\omega-\omega_{0}\right)+\frac{1}{\tau}-\frac{2}{\tau_{e}}}{j\left(\omega-\omega_{0}\right)+\frac{1}{\tau}} \exp (j \omega t)
$$

where $A$ and $\omega$ are the amplitude and the angular frequency of the incident optical carrier, respectively, $\omega_{0}$ is the angular resonance frequency of the ring. The optical field inside the ring decays as a result of the coupling with the bus waveguide and the propagation loss of the doped rib waveguide. The corresponding amplitude decay rates are denoted as $1 / \tau_{\mathrm{e}}$ and $1 / \tau_{1}$, respectively. Thus, the overall decay rate is $1 / \tau=1 / \tau_{\mathrm{e}}+1 / \tau_{\mathrm{l}}$, which is twice the usually referred cavity photon lifetime. The parameter $\mu$ denotes the mutual coupling between the ring and the bus waveguide. According to the power conservation law, the relationship between $\mu$ and $\tau_{\mathrm{e}}$ is $\mu^{2}=2 / \tau_{\mathrm{e}}$ [5].

The embedded PN junction allows the driving RF signal to change the density of free carriers inside silicon waveguide, and subsequently to shift the resonance frequency $\omega_{0}$ and the amplitude decay rate $1 / \tau$ of the ring through the plasma dispersion effect. If a sinusoidal voltage signal in the small signal regime is applied on the device, the modulated optical field through the bus waveguide is deduced as [5]

$$
\begin{aligned}
S_{t}(t) & =A \exp (j \omega t)\left\{\frac{j\left(\omega-\omega_{0}\right)+\frac{1}{\tau}-\frac{2}{\tau_{e}}}{j\left(\omega-\omega_{0}\right)+\frac{1}{\tau}}-\frac{j v_{0} \mu^{2}\left[\frac{\Delta \omega_{0}}{\Delta v_{\mathrm{pn}}}+j \frac{\Delta\left(\frac{1}{\tau}\right)}{\Delta v_{\mathrm{pn}}}\right] f\left(\omega_{1}\right)}{\left[j\left(\omega-\omega_{0}\right)+\frac{1}{\tau}\right]^{2}+\omega_{1}^{2}} \cos \omega_{1} t\right. \\
& \left.-\frac{j v_{0} \mu^{2}\left[\frac{\Delta \omega_{0}}{\Delta v_{\mathrm{pn}}}+j \frac{\Delta\left(\frac{1}{\tau}\right)}{\Delta v_{\mathrm{pn}}}\right] f\left(\omega_{1}\right)}{\left[j\left(\omega-\omega_{0}\right)+\frac{1}{\tau}\right]^{2}+\omega_{1}^{2}} \frac{\omega_{1} \sin \omega_{1} t}{\left[j\left(\omega-\omega_{0}\right)+\frac{1}{\tau}\right]}\right\}
\end{aligned}
$$

where $\Delta \omega_{0} / \Delta \mathrm{v}_{\mathrm{pn}}$ and $\Delta(1 / \tau) / \Delta \mathrm{v}_{\mathrm{pn}}$ denote the change rates of $\omega_{0}$ and $1 / \tau$ with respect to the voltage dropping across the PN junction. If the ring modulator is critical coupled and works at the resonance wavelength, the static transmitted field in the bus waveguide (the first term inside the braces of Eq.2) is zero. Since the photocurrent at the receiver side is proportional to the power of the optical field, i.e., the square of the modulus of Eq. 2, we can get a microwave signal of doubled frequency after the photo-detection. 


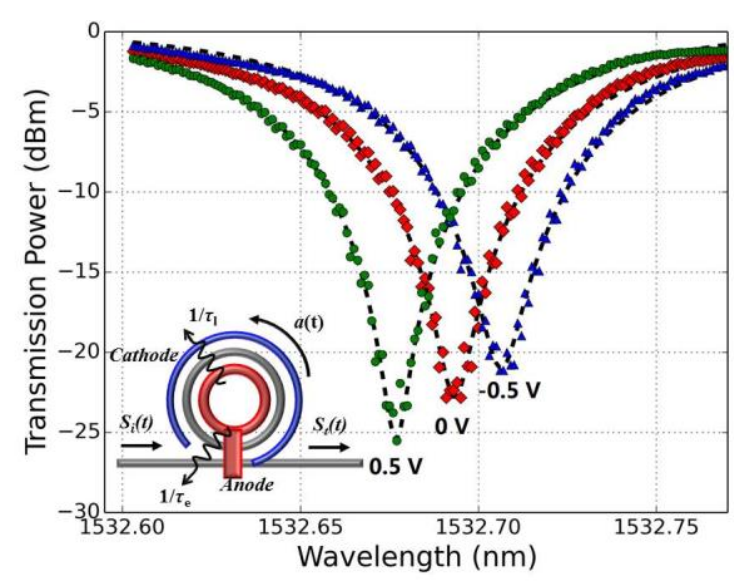

Fig. 1. Transmitted spectra of the ring modulator at different bias voltages. The inset is the schematic diagram of a typical ring modulator

\section{MEASUREMENT RESULTS}

We test a typical carrier-depletion silicon ring modulator of 10 $\mu \mathrm{m}$ radius. The doping concentration of the embedded PN junction is $1 \times 18 / \mathrm{cm}^{2}$. The etching depth and the dimension of the rib waveguide accommodating the PN junction are $150 \mathrm{~nm}$ and $450 \mathrm{~nm} \times 220 \mathrm{~nm}$ respectively. Its transmission spectra at different bias voltages are illustrated in Fig. 1. The extinction of more than $20 \mathrm{~dB}$ at the resonance wavelength indicates that the ring is very close to the critical coupling. To demonstrate the frequency doubling, the input optical carrier of $1 \mathrm{~mW}$ is aligned to the resonance wavelength of the ring. The driving $\mathrm{RF}$ signal has amplitude of $900 \mathrm{mV}$. The modulated optical output from the ring modulator sequentially passes through an EDFA, a tunable filter and finally reaches an electrical spectrum analyzer with an optical head. The frequency conversions from $8 \mathrm{GHz}$ to $16 \mathrm{GHz}$ and from $10 \mathrm{GHz}$ to 20 $\mathrm{GHz}$ are displayed in Figs. 2 (a) and 2 (b), respectively. The suppression ratios between the doubled frequency and the fundamental frequency are $35 \mathrm{~dB}$ for the $16 \mathrm{GHz}$ signal, and $17 \mathrm{~dB}$ for the $20 \mathrm{GHz}$ signal. We attribute the performance degradation as the input frequency rises to the limited modulation bandwidth of this device, which can be improved

(a)

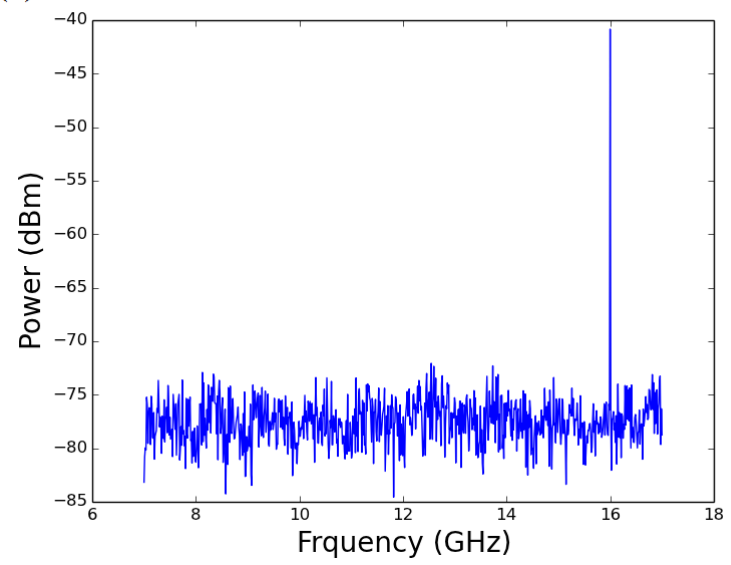

by optimizing the design and the processing condition.

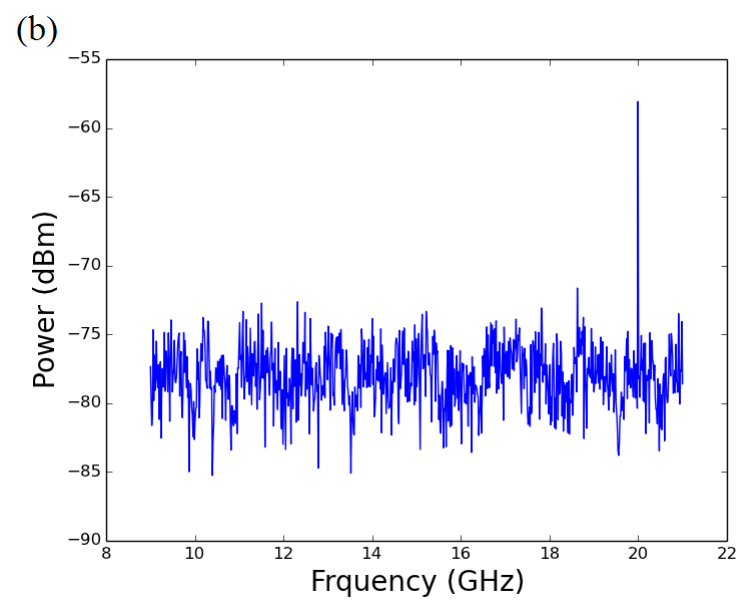

Fig. 2. Measured electrical spectra for (a) $8 \mathrm{GHz}$ to $16 \mathrm{GHz}$ conversion and (b) $10 \mathrm{GHz}$ to $20 \mathrm{GHz}$ conversion.

\section{CONCLUSION}

We propose and experimentally demonstrate a microwave frequency doubling system based on an integrated silicon ring modulator. A $20 \mathrm{GHz}$ signal is obtained from a $10 \mathrm{GHz}$ input signal with $17 \mathrm{dBm}$ suppression ratio. Compared with other techniques, we believe the silicon ring modulator provides a compact, and power/cost efficient candidate for the frequency multiplication in RoF systems.

\section{ACKNOWLEDGMENT}

This work is supported by the Nature Basic Research Program of China (2013CB632105), the National Natural Science Foundations of China (61177055 and 61307074), the Fundamental Research Funds for the Central Universities and China Scholarship Council.

\section{REFERENCE}

[1] F. Liu et al., "Silicon-chip-based frequency quadrupling for optical millimeter-wave signal generation," Proc. SPIE, Optoelectronic Materials and Devices IV, 763105, 2009

[2] Y. Zhao et al., "Simplified optical millimeter-wave generation configuration by frequency quadrupling using two cascaded MachZehnder modulators", Optics Letters, vol. 34(21), pp. 3250-3252, 2009

[3] L. Gao et al., "Photonic-assisted microwave frequency multiplication with a tunable multiplication factor", Optics Letters, vol. 38(21), pp. 4487-4490, 2013

[4] G. Qi, etal, "Generation and distribution of a wide-band continuously tunable millimeter-wave signal with an optical external modulation technique," IEEE Trans. Microwave Theory Techno., vol. 53(10), 30903097, 2005

[5] H. Yu, "Trade-off between optial modulation amplitude and modulation bandwidth of silicon micro-ring modulators," Submit to Opt. Express, 2014 